This item was submitted to Loughborough's Research Repository by the author.

Items in Figshare are protected by copyright, with all rights reserved, unless otherwise indicated.

\title{
Simulations of delamination in CFRP laminates: effect of microstructural randomness
}

PLEASE CITE THE PUBLISHED VERSION

PUBLISHER

(C) Elsevier

VERSION

AM (Accepted Manuscript)

LICENCE

CC BY-NC-ND 4.0

\section{REPOSITORY RECORD}

Khokhar, Zahid R., lan A. Ashcroft, and Vadim V. Silberschmidt. 2019. "Simulations of Delamination in CFRP Laminates: Effect of Microstructural Randomness”. figshare. https://hdl.handle.net/2134/5254. 
This item was submitted to Loughborough's Institutional Repository (https://dspace.lboro.ac.uk/) by the author and is made available under the following Creative Commons Licence conditions.

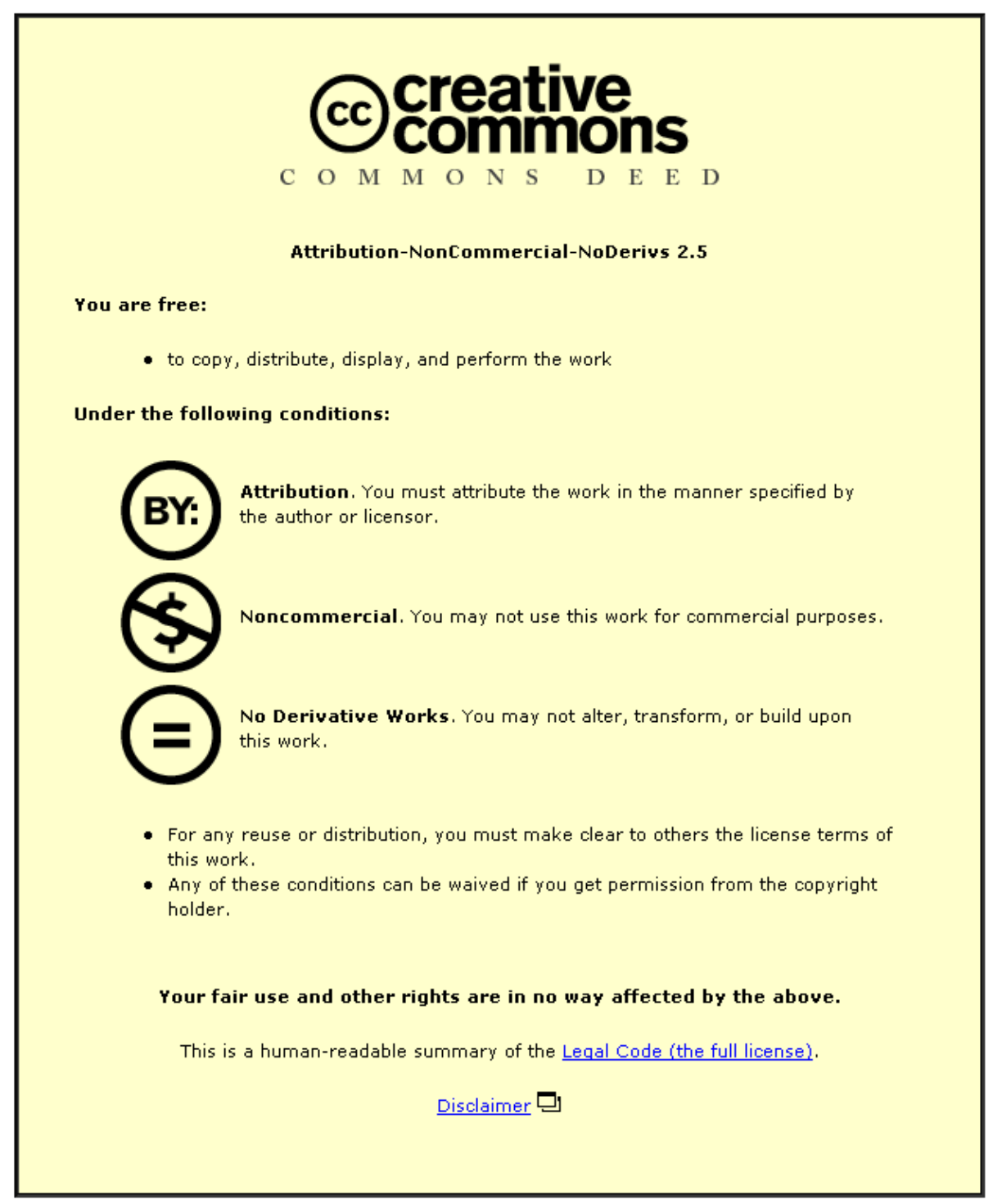

For the full text of this licence, please go to: http://creativecommons.org/licenses/by-nc-nd/2.5/ 


\title{
SIMULATIONS OF DELAMINATION IN CFRP LAMINATES:
}

\section{EFFECT OF MICROSTRUCTURAL RANDOMNESS}

\author{
Zahid R. Khokhar, Ian A. Ashcroft and Vadim V. Silberschmidt \\ Wolfson School of Mechanical and Manufacturing Engineering, Loughborough \\ University, Loughborough, Leicestershire, LE11 3TU, UK
}

\begin{abstract}
Due to their high specific strength and stiffness, fibre-reinforced composite materials are being increasingly used in structural applications where a high level of performance is important (e.g. aerospace, automotive, offshore structures, etc.). Performance in service of these composites is affected by multi-mechanism damage evolution under loading and environmental conditions. For instance, carbon fibre-reinforced laminates demonstrate a wide spectrum of failure mechanisms such as matrix cracking and delamination. These damage mechanisms can result in significant deterioration of the residual stiffness and load-bearing capacity of composite components and should be thoroughly investigated. The delamination failure mechanism is studied in this paper for a double cantilever beam (DCB) loaded in Mode I. Several sensitivity studies are performed to analyse the effects of mesh density and of parameters of the cohesive law on the character of damage propagation in laminates. The microstructural randomness of laminates that is responsible for non-uniform distributions of stresses in them even under uniform loading conditions is accounted for in the model. The random properties are introduced with the use of Weibull's two-parameter probability density function. Several statistical realisations are carried out which show that the effect of microstructure could significantly affect the macroscopic response emphasizing the need to account for
\end{abstract}


microstructural randomness for accurate predictions of load-carrying capacity of laminate composite structures.

Keywords: CFRP laminates; Delamination; Cohesive zone modelling; Microstructural randomness; Weibull’s distribution.

\section{Introduction}

\subsection{Delamination in laminates: Effect of Microstructure}

Failure in composite structures is often caused by the development of different damage mechanisms induced in local zones. The particular damage modes depend upon the type of loading, the lay-up and stacking sequence of the composite. For effective predictive capabilities, all mechanisms of failure must be taken into account. In fibre-reinforced laminates delamination between plies is one of the most common types of damage due to relatively low interlaminar strengths of such composites. The nature of the delamination process in them is complex presupposing the use of advanced FE modelling techniques for its analysis. The simulation using the failure analysis techniques should have the

capability of predicting the initiation, size and propagation of delamination. Another important factor affecting this damage mode is the effect of microstructural randomness on the delamination behaviour in CFRP laminates.

In most micromechanical analyses of fibre-reinforced composite materials, an assumption of spatial periodicity of their properties is employed: it is considered that the material has an ordered (deterministic) distribution of fibres. Such periodic distributions are usually assumed, based typically, on a square or hexagonal layout used in a representative volume element (RVE). Employing this periodicity assumption, effective properties for composite materials can be computed, and various homogenization 
theories and two-scale methods have been developed under this assumption [1]. These theories have been applied for the simulation of fibre-reinforced composite materials with linear [2] and non-linear behaviours [3]. Still, in reality microstructure of fibrereinforced composites is far from being ordered since fibres are usually randomly distributed in the matrix. This can be easily noticed even with simple optic microscopy as shown in Fig. 1.

Wang et al. [4] were among the first to consider the location of matrix cracks as a statistical quantity in their models. They presented a stochastic simulation model for the growth of multiple matrix cracks in cross-ply laminates subjected to both static and fatigue loads. Fukunaga et al. [5] combined the shear-lag analysis with stochastic analyses to study progressive matrix cracking in carbon fibre cross-ply laminates. Baxevanakis et al. [6] demonstrated a high level of spatial non-uniformity for a composite by applying the image analysis technique to cross-sectional areas of T300/914 specimens. In this composite with an average volume fraction of fibres 55.9\%, the local minimum and maximum levels of the observed volume fractions were $15 \%$ and $85 \%$, respectively. Such dispersion and scatter undermine the use of schemes, based on ideal periodic or quasi-periodic arrangements of fibres in estimation of the effective properties and, especially, failure parameters of such composites. Bulsara et al. [7] presented their study of the RVE size appropriate for characterization of initiation of damage under a tensile load normal to fibres in a unidirectional composite. That work revealed that the scatter among random realisations of RVE varies with the RVE size and the appropriate size of the RVE should be linked to the lowest scatter. Silberschmidt has established that cross-ply carbon-epoxy laminates demonstrate a considerable extent of randomness in distributions of transverse cracks in $90^{\circ}$ layers [8] and studied the effect of 
microstructural randomness on the distribution of matrix cracks in these laminates [9]. Solutions for sets of cracks with minimum and maximum spacings were performed, which in a majority of cases provided lower and upper bounds, respectively, for macroscopic properties of laminates. It was concluded that the behaviour of specimens with multiple matrix cracks cannot be always reduced to analysis of a single traditional unit cell as an area limited by two neighbouring cracks under the assumption of equal crack spacing. Trias et al. [10] showed by comparing stress and strain distributions obtained with periodic and random models for a carbon fibre-reinforced polymer that periodic models can be used to assess effective properties but random ones must be considered for simulations of local phenomena such as damage accumulation or matrix cracking. Silberschmidt [11] further contributed to this topic by presenting a lattice model to study damage and fracture evolution in laminates, linking microstructural randomness with macroscopic properties and demonstrating that a random character of the fibres' distribution results in fluctuations of local elastic moduli in composites, the bounds of which depend on the characteristic length scale.

Hence, previous works have vividly demonstrated the microscopically random nature of CFRP laminates. This paper emphasizes the fact that this spatial non-uniformity should be directly taken into account while addressing damage scenarios. A delamination failure mechanism in a DCB specimen is modelled with an account of the microstructural randomness. The methodology employed to analyse is the cohesive zone modelling, a brief overview of which follows.

\subsection{Cohesive zone modelling}

The analysis of fracture development has changed considerably in the last few decades. 
Instead of traditional approaches of fracture mechanics, various predictive schemes such as cohesive zone models (CZMs) or traction-separation models are now increasingly being used, especially in numerical simulations. CZMs are established as relatively simple methods, which are easier to implement in finite-element (FE) codes and, as a result, CZ elements are now available in various commercial FE packages.

One of the first studies into cohesive zones was performed by Barenblatt [12], who formulated the fundamental idea for a CZM, investigated the fracture of quasi-brittle materials by defining the traction along the crack path as a function of the distance from a crack tip along the crack front. Needleman $[13,14]$ was one of the first to use polynomial and exponential types of traction-separation relations to simulate the particle debonding in metal matrices. An exponential fit was used for a normal traction while a trigonometric one for a shear traction. To sum up, cohesive zone modelling were found very useful in tackling problems of non-linear fracture mechanics.

This paper includes implementation of the cohesive zone modelling technique based on a bilinear traction-separation law. The mechanical response of the interface between plies which is a potential site for delamination in laminates is described by constitutive equations, which relate the applied stress $\sigma$, normal to the interface, to the relative displacement in the same direction at the interface $\delta$ given by a bilinear relationship. This relationship can be subdivided into three main parts (Fig. 2) given by the following constitutive equations [15]:

Elastic part: $\quad \delta<\delta_{\text {c }} \quad ; \quad \sigma=K \delta$;

Softening part: $\quad \delta_{\mathrm{c}}<\delta<\delta_{\mathrm{f}} ; \sigma=(1-D) K \delta$;

De-cohesion part: $\quad \delta>\delta_{\mathrm{f}} ; \sigma=0$, 
where $D$ is a damage characterizing variable, which has a zero value in the virgin (undamaged) state and attains a value of one when the material is fully damaged; $K$ is the initial stiffness; $\delta_{\text {c }}$ is the critical displacement; $\delta_{\mathrm{f}}$ is the maximum displacement; and $\sigma$ is the tripping traction.

The area under the curve formed by these lines in Fig. 2 is the fracture energy $G_{F}$ for a particular mode.

As a first stage of the analysis of two-mode damage, i.e. matrix cracking and delamination in CFRP laminates, this paper aims to analyze the delamination in unidirectional laminates. In Section 2 of the paper, numerical analysis of delamination of a DCB is carried out assuming a homogenized material with spatially uniform properties. This is a standard scheme used in traditional CZM-based approaches. The subsequent part of the analysis presented in Section 3 focuses on the effect of microstructural randomness in the laminates. This is achieved by employing various methods to introduce into FE models random fracture properties along the length of cohesive zone. This means a transition to a stochastic cohesive zone model. FE software MSc Marc is used to simulate delamination in the DCB for all the methods.

\section{Delamination in CFRPs: Cohesive zone modelling in a DCB specimen}

The delamination failure in fibre-reinforced composites is frequently studied [16] by employing the double-cantilever beam specimen. A DCB test is traditionally utilized to determine mode I (tensile-opening) interlaminar fracture toughness $G_{\text {IC }}$ of the material under consideration. This test, based on a special type of linear-elastic fracture-mechanics 
specimen, is incorporated in several international test standards; this study is based on the standard test method given in ISO 15024 [17] .

The test, simulated in the paper, was performed with a specimen made of a unidirectional carbon fibre-reinforced laminate with an epoxy matrix. The volume fraction of the thermosetting matrix was nominally 35\% and the carbon fibres were T400 [18]. The specimen was $125 \mathrm{~mm}$ long, $20 \mathrm{~mm}$ wide, with twenty four $0.125 \mathrm{~mm}$ thick plies (12 on each side of delamination modelled as a layer of cohesive elements), and with an initial crack length of $30 \mathrm{~mm}$. The properties of the material are given in Table 1.

A delamination growth was simulated with finite elements by placing interfacial decohesion elements between the composite layers. A decohesion failure criterion that combines aspects of strength-based analysis and fracture mechanics is used to simulate debonding by reducing the stiffness of the softening element.

A two-dimensional model was developed for the study using finite element software MSc Marc and is shown in the Fig. 3. The CFRP material is presented as a continuum that has anisotropic properties while the response of interface to the external load is governed by a bilinear traction-separation law (Fig. 2).

\subsection{Calculation of cohesive parameters}

Parameters of the cohesive law are given in Table 2 with their magnitudes used in simulations calculated from the relations taken from the work of Turon et al. [19]. In order to model progressive damage and failure using the CZM approach, a pre-defined crack path has to be provided as input to the model. The cohesive zone model simulates the macroscopic damage along this path by specification of a traction-separation response between initially coincident nodes on either side of the pre-defined crack. For DCB 
specimens the path is naturally defined as the interface between composite plies containing the initial crack. The analysis is performed for a displacement-control method. A displacement $u$ is applied at the ends of the double cantilever beam as shown in Fig. 3. The area occupied by the composite is meshed using Element type 11, which is a four-node, iso-parametric, arbitrary quadrilateral element used for the plane-strain state. It is a fully-integrated element. To improve simulation of the bending behaviour, incompatible modes are used with the 'assumed strain' formulation available in MSC.Marc. Large deformations are taken into account in this study.

On the other hand, to discretize the cohesive zone, Element type 186 is used to simulate the onset and progress of delamination. It is a mechanical four-node planar interface element. The edge length used for meshing at this stage of analysis was $0.15 \mathrm{~mm}$. The cohesive elements behave as stiff springs until a critical (maximum) traction is reached. The spring unloads progressively after this point, and this unloading process dissipates energy. The nodes start to separate upon unloading and then finally de-bond totally. In such a way, the crack propagation and the failure evolution can be simulated. The parameters that govern the traction-separation law are the maximum traction $\left(\sigma_{\max }\right)$ and the energy of separation per unit area $(G)$. When introducing cohesive elements into the FE model it is important that their thickness direction is defined correctly. It should point in the direction of the crack opening , i.e., it is vertical in our case.

A calculated load-displacement plot for the DCB is given in Fig. 4, which gives a load of 83.8 $\mathrm{N}$ for onset of damage (point $\mathrm{A}$ on the figure). The value of the corresponding displacement $u$ at this load is found out to be $4.15 \mathrm{~mm}$.

The following section is dedicated to sensitivity analyses of the numerical solution to various parameters. 


\subsection{Sensitivity analyses}

Several sensitivity studies were performed to analyse the effect of mesh density and initial stiffness of the cohesive zone law on the overall damage behaviour of CFRP laminates.

\section{$\underline{\text { Sensitivity to initial stiffness }}$}

In order to analyse the sensitivity of the delamination analysis to the interface stiffness, the double cantilever beams was simulated for various levels of that parameters with the same mesh size. The edge length of elements used for all these cases was $0.2 \mathrm{~mm}$. The calculated load-displacement plots are presented in Fig. 5.

Obviously, the curves for higher values of interface stiffness demonstrate a stable response. Lower values, e.g. $10^{4} \mathrm{~N} / \mathrm{mm}^{3}$, do not provide a stiff connection between two neighbouring layers resulting in unstable behaviour. As a result, the value for initial stiffness of $2.283 \times 10^{6} \mathrm{~N} / \mathrm{mm}^{3}$, calculated based on [19], was chosen for the traction-separation law and used throughout this work.

\section{$\underline{\text { Sensitivity to mesh size }}$}

A certain mesh bias is experienced by cohesive zone models in their numerical implementation. Constitutive properties of these elements describe the evolution of cohesive traction (stress) as the interface is being opened (opening displacement). The direction of crack propagation strongly depends on the presence (or absence) of cohesive zone elements, since the crack path can only follow these elements. The extent of crack growth along the prescribed path defined by CZ elements depends, in its turn, on the size of these elements. Obviously, the problem of mesh-dependency can be reduced by 
refining the mesh. Still, such refinement can be prohibitive since it needs a significant increase in computational efforts.

To highlight such mesh sensitivity of cohesive problems, several simulations were carried out with different mesh sizes from $0.5 \mathrm{~mm}$ to $0.025 \mathrm{~mm}$. The calculated graphs (Fig. 6) show the respective variations in the critical values of load and opening displacements.

Results of simulations vividly demonstrate that an increase in the mesh density leads to the decrease in levels of the critical load and opening displacement. Finally, the mesh size of $0.15 \mathrm{~mm}$ was chosen to reduce computational efforts since it provided a satisfactory level of detailedness in numerical studies. Though the difference between magnitudes of critical loads for the chosen mesh of $0.15 \mathrm{~mm}$ and the finest one (i.e. $0.025 \mathrm{~mm}$ ) is $11.4 \%$, the former was chosen for all analysed models. The main reason for this was a demand of consistency and comparability of results, obtained with current 2D finite-element formulations, and those of the next stage of research, when 3D discretization will be introduced. Even solution of presented 2D problems required the use of a high performance computing multi-nod cluster with a large shared memory; the smaller mesh size would make analysis of 3D cases computationally prohibitive.

\section{Effect of microstructural randomness on delamination in laminates}

As mentioned earlier, CFRP composites exhibit randomness at the level of their microstructure that affects their mechanical properties and hence the damage initiation and evolution process. The next stage of our study is linked with an account of this microstructural randomness that is responsible for non-uniform distributions of stresses in 
real composites even under macroscopically uniform external loading. The effect of this randomness on the delamination behaviour of unidirectional composites is analysed by means of introduction of random fracture properties within the layer of cohesive elements in a DCB specimen. Two different methods, described below, were employed for that purpose. A value of half scatter of $50 \%$ for fracture energy $G_{\mathrm{F}}$ was selected as a starting point to underline the effect of the material's stochastic nature on the course of damage initiation and propagation. This is achieved by defining spatially random levels of fracture energy in the cohesive zone model.

Figure 7 shows the effect of variation of fracture energy on bilinear traction-separation curves with values of critical and maximum opening displacements remaining constant. For the same values of opening displacements, by changing the value of fracture energy from $G_{\mathrm{F}}$ to $0.5 G_{\mathrm{F}}$, the values of tripping traction and initial stiffness of cohesive law both decrease (Fig. 7). An opposite development takes place when the value of fracture energy changes from $G_{\mathrm{F}}$ to $1.5 G_{\mathrm{F}}$.

The same mesh density of $0.15 \mathrm{~mm}$ was used in discretization of the model; all the loading parameters and boundary conditions were the same in all cases of different schemes.

\subsection{Method 1}

In the first scheme, variation of fracture energy is introduced along the layer of cohesive elements in the double cantilever beam by using three different levels of properties, i.e., $0.5 G_{\mathrm{F}}, G_{\mathrm{F}}$ and $1.5 G_{\mathrm{F}}$ (in all simulations $G_{\mathrm{F}}=0.257 \mathrm{~kJ} / \mathrm{m}^{2}$ ). The cohesive layer between the continuum elements is meshed with cohesive elements having zero thickness. Different elements in the cohesive elements layer are assigned different levels of 
properties, so that fracture energy levels in adjoining elements differ by not more than $50 \%$. The length of cohesive zone was $90 \mathrm{~mm}$. The results in the form of load-displacement and damage plots were obtained together with the distribution of damage $D$ along the layer of cohesive zone elements.

As the DCB specimen opens, the type of neighbouring elements affects the delamination growth. For instance, a stronger part of the cohesive layer would resist opening stronger and hence increases the force. A load-displacement plot of the analysis is shown in Fig. 8. The post-critical behaviour of the specimen after the onset of delamination is presented in Fig. 8(b) at the enlarged scale making evident its non-monotonous behaviour.

To further highlight the effect of spatial non-uniformity on the damage of CFRP laminates, various statistical realisations for the same type of randomness were used to study the distribution of damage along the length of cohesive zone and the effect of spatial non-uniformity of local fracture properties. The results obtained for different realisations are shown in Fig. 9. These diagrams demonstrate considerably different levels of damage at same locations of the delamination zone. The delamination zones developed in front of the fully separated parts of DCB are the secondary delamination zones that are often observed in real composites.

Numerical simulations based on this method revealed a relatively low level of scatter in the obtained results. For instance, the scatter of the critical load is below $2 \%$.The differences in the load-carrying capacity - though small - and in the damage initiation and propagation due to the introduction of microstructural randomness in the laminates under investigation call for the treatment of the problem in a more sophisticated way so other methods were developed. 


\subsection{Method 2}

In this method, the fracture energy in elements along the cohesive layer was introduced based on a two-parameter Weibull's distribution. This is achieved at first by representing the fracture energy of cohesive elements by a distribution with a constant probability density for the same scatter width $\left[G_{\mathrm{F}}{ }^{\min }, G_{\mathrm{F}}{ }^{\max }\right.$ ] as in Method 1 , so a set of magnitudes between the two extreme values $\left(0.5 G_{\mathrm{F}}\right.$ and $\left.1.5 G_{\mathrm{F}}\right)$ was produced using the random number generator. Those random numbers were transformed to comply with the Weibull's distribution, and a set of random energy magnitudes were obtained based on that distribution (shown in Fig. 10); the generated data is presented in Fig. 11. The number of data points $N$ in the set was equal to the number of nodes in the cohesive layer of the finite element model, to which it were assigned. The same average magnitude of the fracture energy $\left\langle G_{\mathrm{F}}\right\rangle=\frac{1}{N} \sum_{i=1}^{N} G_{\mathrm{F} i}$, equal to the one of the uniform model (i.e. $G_{\mathrm{F}}=$ $0.257 \mathrm{~kJ} / \mathrm{m}^{2}$ ), was used in each statistical realisation in both Methods 1 and 2 . There were small deviations from this magnitude in Method 2 only due to the discretization of the Weibull's distribution function. It was checked that the latter did not cause a systematic change in the calculated load-displacement relations obtained for different statistical realisations.

In order to study the effect of randomness in material properties on the critical load and critical opening displacement, a series of simulations was carried out based on several statistical realisations for the Weibull's distribution with the same parameters. Different sets of random numbers were generated for various seeds. The obtained results for levels of critical load and critical opening displacement show a large spread; both higher and lower values of critical load are obtained compared to the uniform distribution model 
(Figs 12 (a) and 12 (b), respectively).

To conclude, these results show that the effect of microstructure could significantly affect the macroscopic response of the DCB to external loading. Instead of a single level of the critical force for the critical displacement, a band of magnitudes is obtained that includes a deterministic case. Obviously, the most concerning cases are those that have the critical load below that for uniform material properties. Hence, there is a need to account for microstructural randomness for accurate predictions of load-carrying capacities of laminated composite structures.

\section{Conclusions}

The underlying objective of this study was to model the delamination failure mechanism in laminated composites in order to highlight the effect of microstructural randomness of such laminates on delamination. A standard DCB specimen was analysed with the use of cohesive zone elements presenting the delamination zone. The results for the model with uniform and random fracture properties were obtained, and the comparison of results shows the variation in the overall force in the DCB specimen as well as the damage variation due to this introduction of randomness. The obtained data for forces and corresponding displacements demonstrates a difference in the results of the two types of models, i.e. the one assuming uniform microstructure with constant material properties along the entire cohesive layer, and the other, random models, with a spatial variation/scatter in fracture energy and same bounds of the scatter applied. Even for 10 statistical realisations the width of the scatter for the critical load was more than $40 \%$ (in terms of the one for the uniform properties). These results emphasise the importance of taking into account the microstructural randomness exhibited by CFRP laminates as 
considerable variations in load carrying capacity were observed. There are realisations that give the critical values, exceeding those obtained for uniform properties; in such cases the structure is deemed to be conservatively designed. But, importantly, some statistical realisations predicted significantly lower values of the critical load compared to the case with uniform properties. These cases specifically emphasise the need to consider the microstructural randomness by applying the spatial non-uniformity of properties exhibited by the CFRP laminates when addressing damage scenarios.

The focus of our future research will be on interaction of matrix cracking and delamination modes in carbon fibre-reinforced laminates using suggested stochastic cohesive zone elements.

\section{References}

[1] P. Suquet, Elements of homogenization for inelastic solid mechanics, Homogenization Techniques for Composite Media, Springer-Verlag, (1987).

[2] C.T. Sun and R.S. Vaidya, Prediction of composite properties from a representative volume element, Composites Science and Technology, 56 (1996) 171-179.

[3] S. Li, General unit cells for micromechanical analyses of unidirectional composites, Composites Part A: Applied Science and Manufacturing, 32 (2001) 815-826.

[4] A.S.D. Wang, P.C. Chou, and S.C. Lei, A stochastic model for the growth of matrix cracks in composite materials, Journal of Composite Materials, 18 (1984) 239-254.

[5] H. Fukunaga, C. Tsu-Wei, P.W.M. Peters, and K. Schulte, Probabilistic failure 
strength analysis of graphite epoxy cross ply laminates, Journal of Composite Materials, 18 (1984) 339-356.

[6] C. Baxevanakis, D. Jeulin, and J. Renard, Fracture statistics of a unidirectional composite, International Journal of Fracture, 73 (1995) 73-149.

[7] V.N. Bulsara, R. Talreja, and J. Qu, Damage initiation under transverse loading of unidirectional composites with arbitrarily distributed fibres, Composites Science and Technology, 59 (1999) 673-682.

[8] V.V. Silberschmidt, Scaling and multifractal character of matrix cracking in carbon fibre-reinforced cross-ply laminates, Mechanics of composite materials and structures, 2 (1995) 243-255.

[9] V.V. Silberschmidt, Matrix cracking in cross-ply laminates: effect of randomness, Composites Part A: Applied Science and Manufacturing, 36 (2005) 129-135.

[10] D. Trias, J. Costa, J.A. Mayugo, and J.E. Hurtado, Random models versus periodic models for fibre reinforced composites, Computational Materials Science, 38 (2006) 316-324.

[11] V.V. Silberschmidt, Effect of micro-randomness on macroscopic properties and fracture of laminates, Journal of Material Science, 41 (2006) 6768-6776.

[12] G.I. Barenblatt, The mathematical theory of equilibrium cracks in brittle fracture, Advances in Applied Mechanics, 7 (1962) 55-129.

[13] A. Needleman, A continuum odel for void nucleation by inclusion debonding, Journal of Applied Mechanics, 54 (1987) 525-531.

[14] A. Needleman, An analysis of tensile decohesion along an interface, Journal of the Mechanics and Physics of Solids, 38 (1990a) 289-324.

[15] M. Meo and E. Thieulot, Delamination modelling in a double cantilever beam, 
Composite Structures, 71 (2005) 429-434.

[16] B.R.K. Blackman, H. Hadavinia, A.J. Kinloch, and J.G. Williams, The use of a cohesive zone model to study the fracture of fibre composites and adhesively-bonded joints, International Journal of Fracture 119 (2003) 25-46.

[17] Fibre-reinforced plastic composites - Determination of mode I interlaminar fracture toughness, GIC, for unidirectionally reinforced materials, ISO 15024 (2001).

[18] B.R.K. Blackman, J.P. Dear, A.J. Kinloch, H. Macgillivray, Y. Wang, W.J. G., and P. Yayla, The failure of fibre composites and adhesively bonded fibre composites under high rates of test, Journal of Materials Science, 30 (1995) 5885-5900.

[19] A. Turon, C.G. Davila, P.P. Camanho, and J. Costa, An engineering solution for using coarse meshes in the simulation of delamination with cohesive zone models, NASA Technical Memorandum, (2005). 


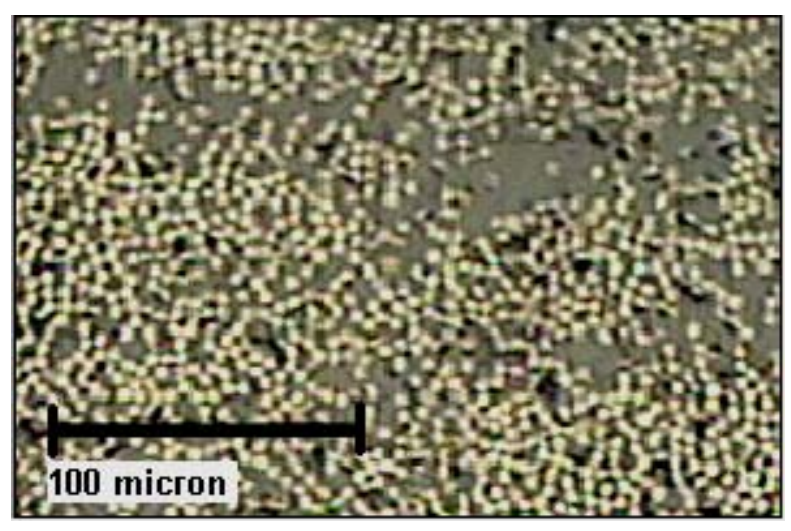

Fig.1 Transversal cross-section of a carbon fibre-reinforced unidirectional laminate demonstrating spatial randomness in distribution of fibres

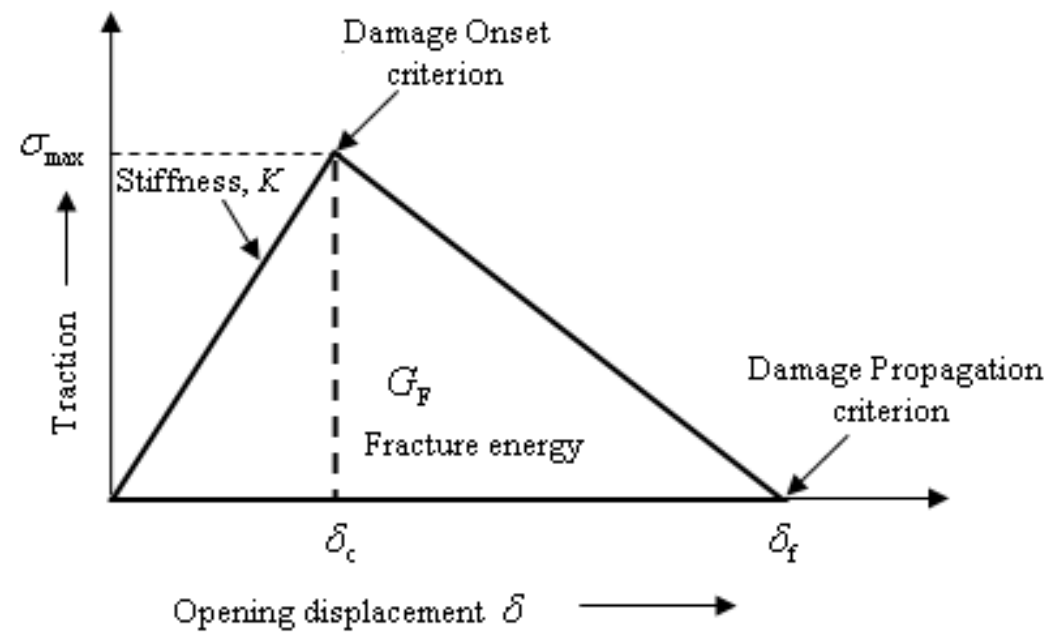

Fig. 2 Bilinear traction-separation law (after [15]) 


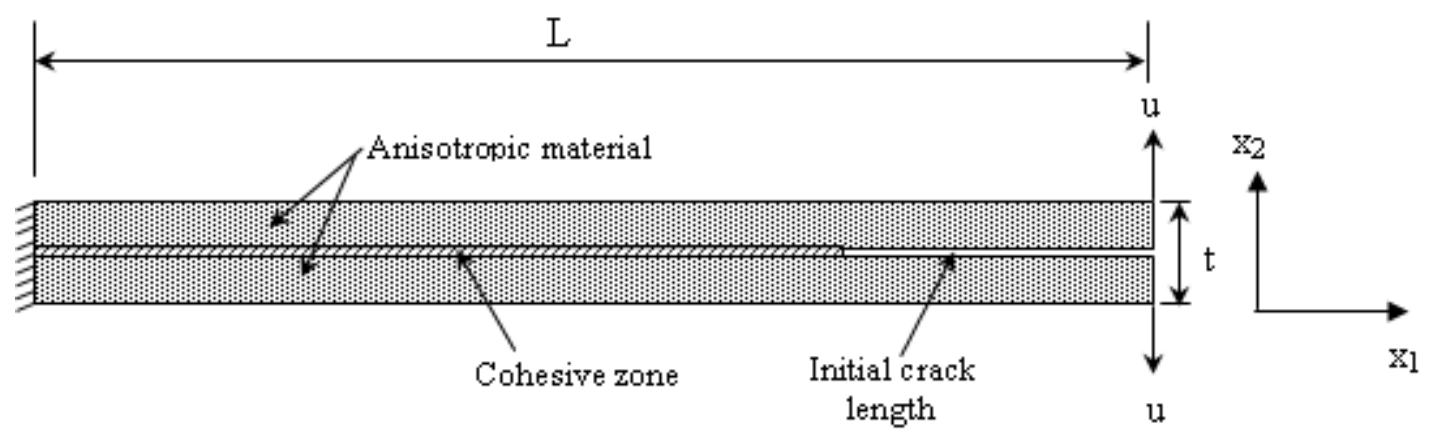

Fig. 3 Double cantilever beam model

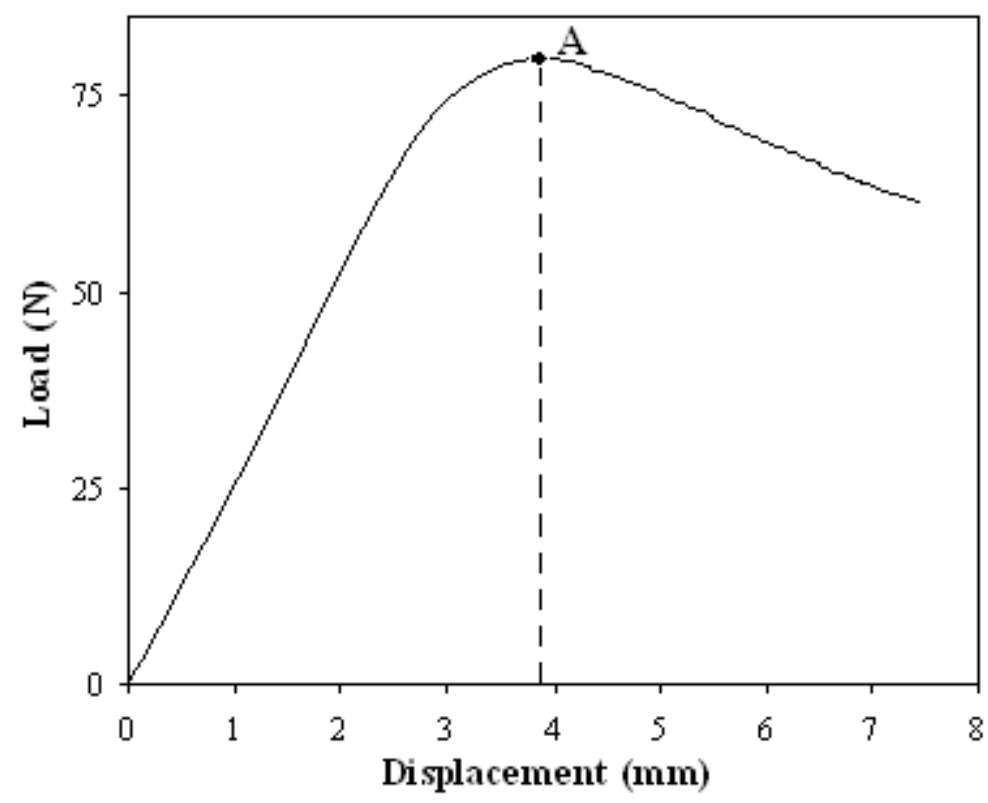

Fig. 4 Calculated load-displacement diagram for a DCB with uniform cohesive properties 


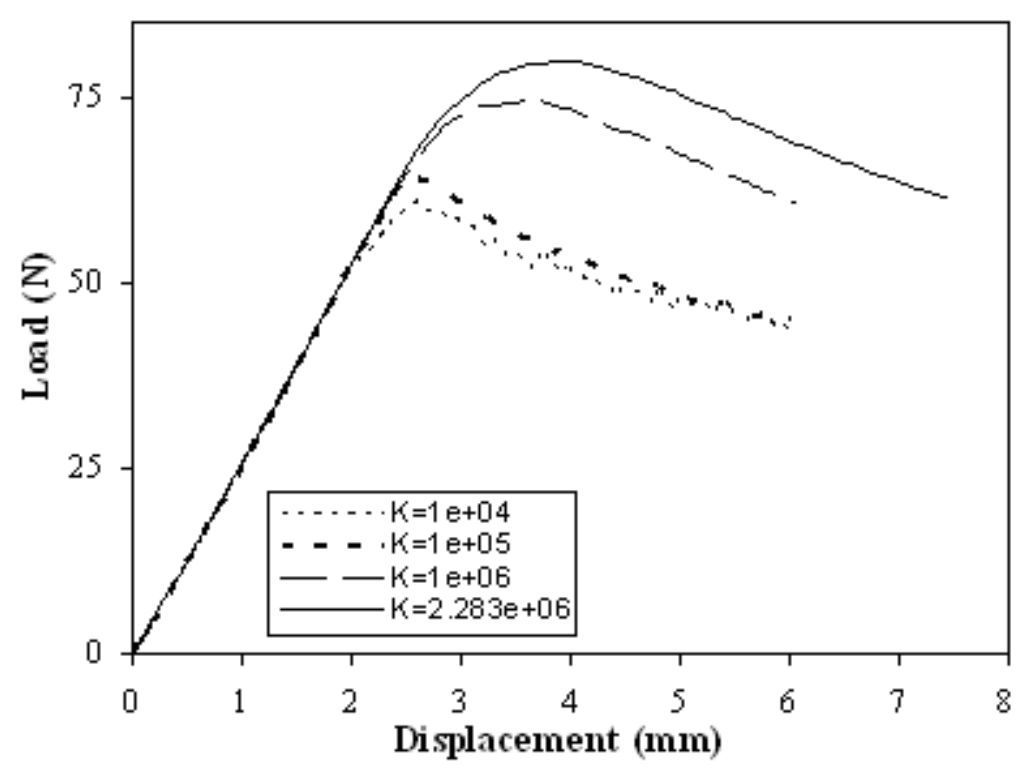

Fig. 5 Sensitivity of load-displacement plot to initial stiffness

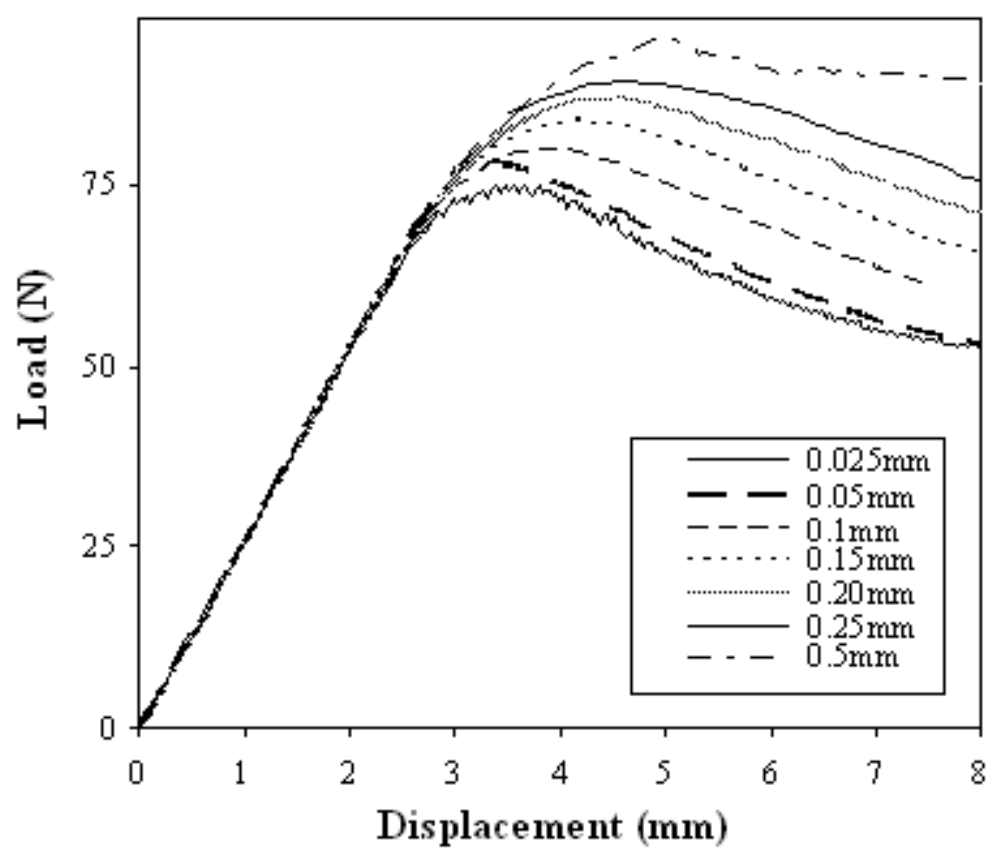

Fig. 6 Load-displacement plot for various mesh densities 


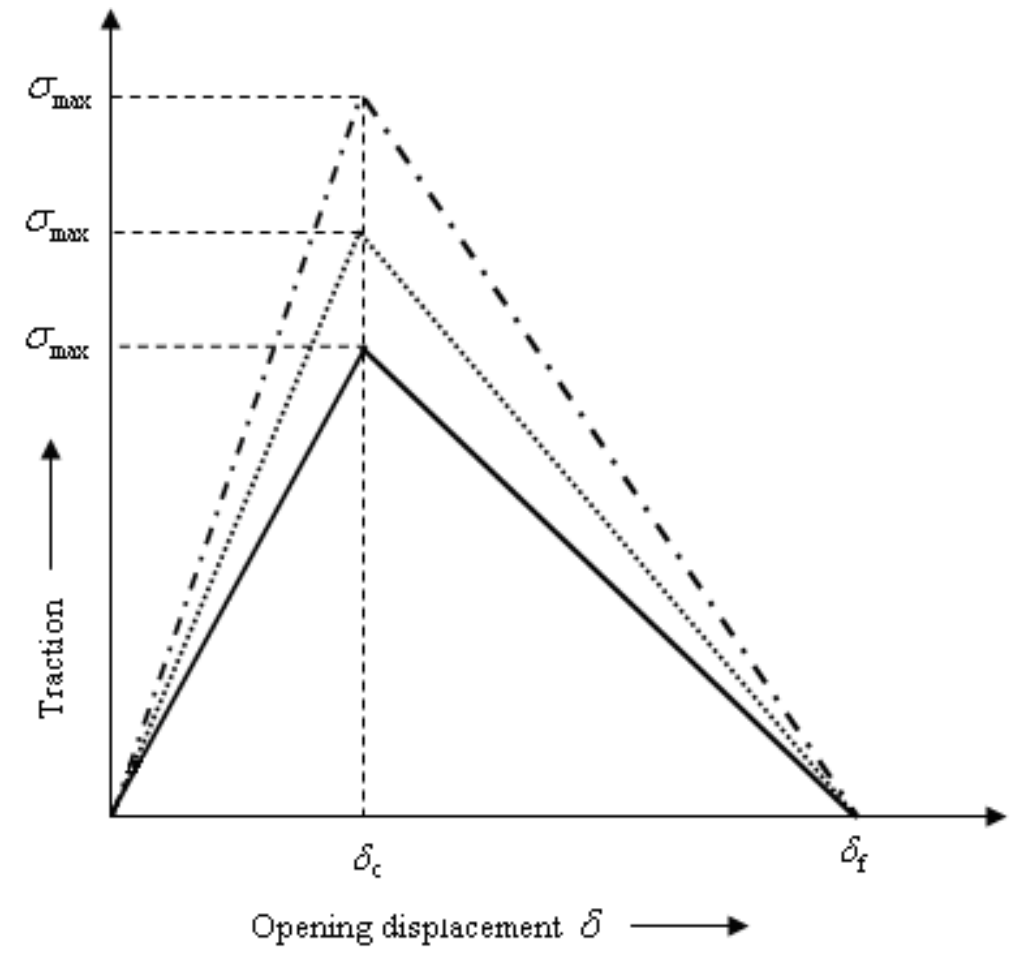

Fig. 7 Cohesive law with variation of $G_{F}$ for the same opening displacement values

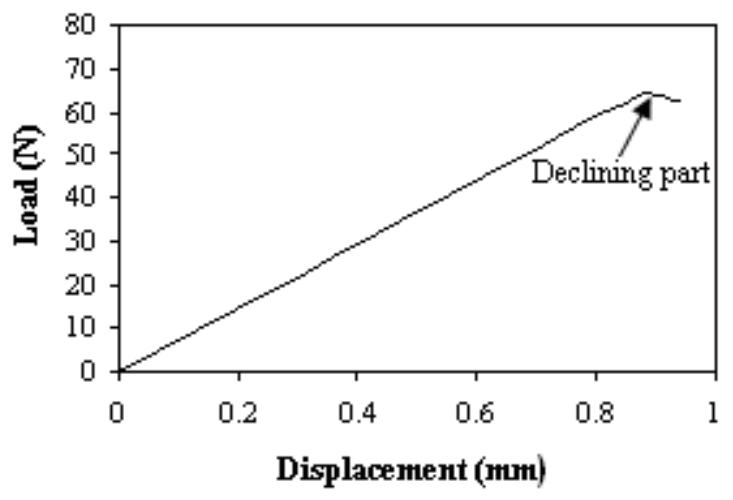

(a)

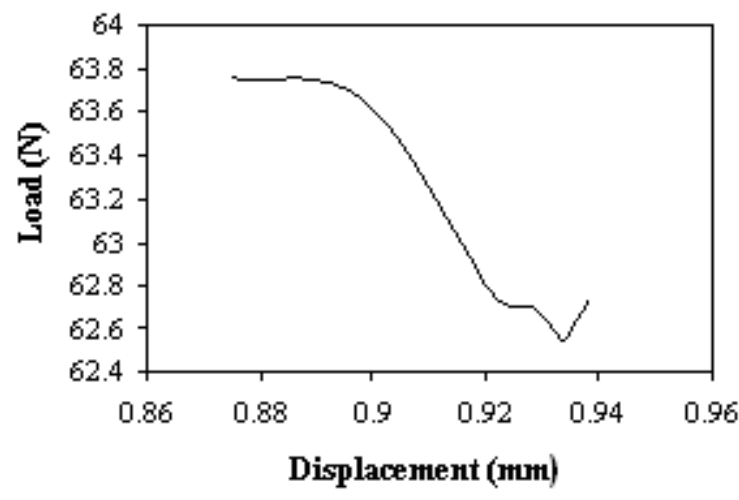

(b)

Fig. 8 Load-displacement diagram for DCB with varying fracture energy within the cohesive layer (a) and its declining part (b) 


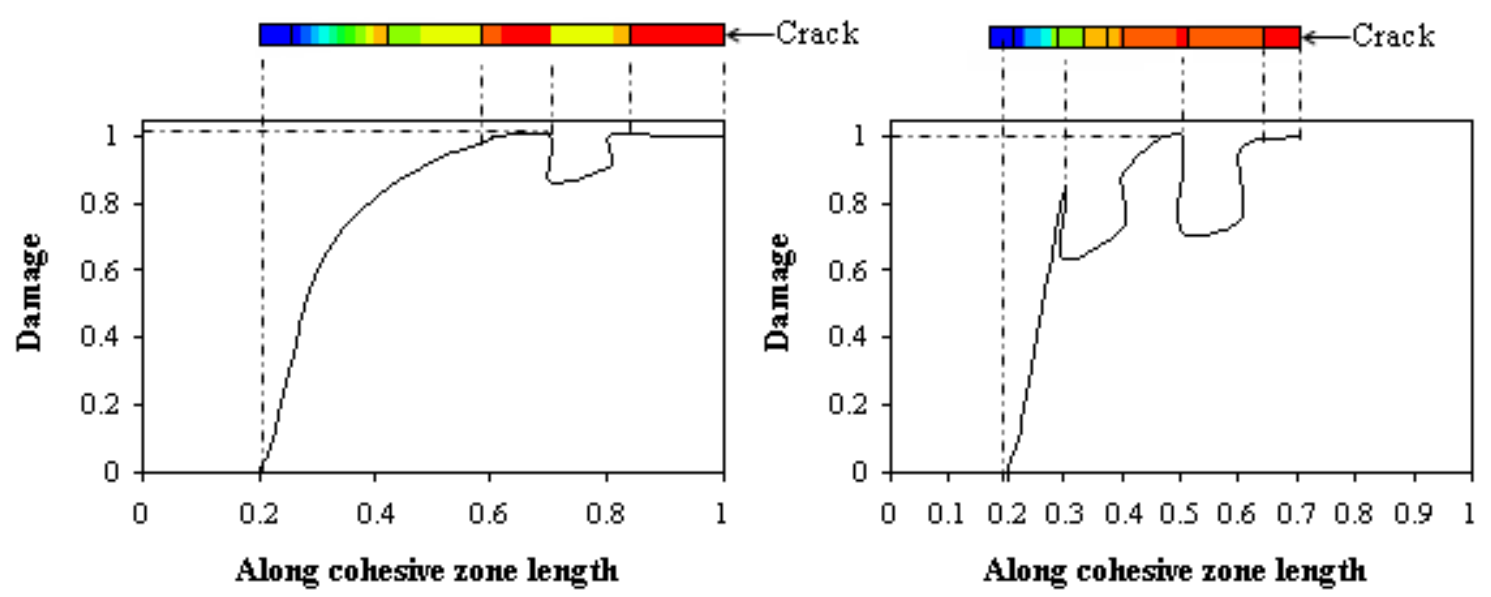

Fig. 9 Variation in damage along the cohesive layer for two statistical realisations of local fracture energy levels (Method 1)

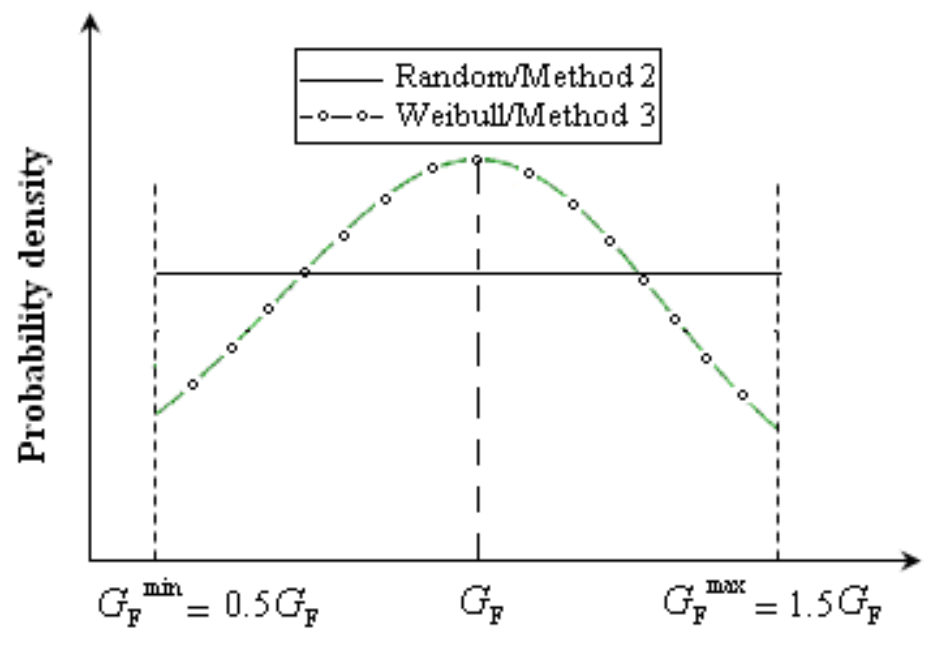

Fracture energy

Fig. 10 Two probability density functions used in simulations with random levels of fracture energy 


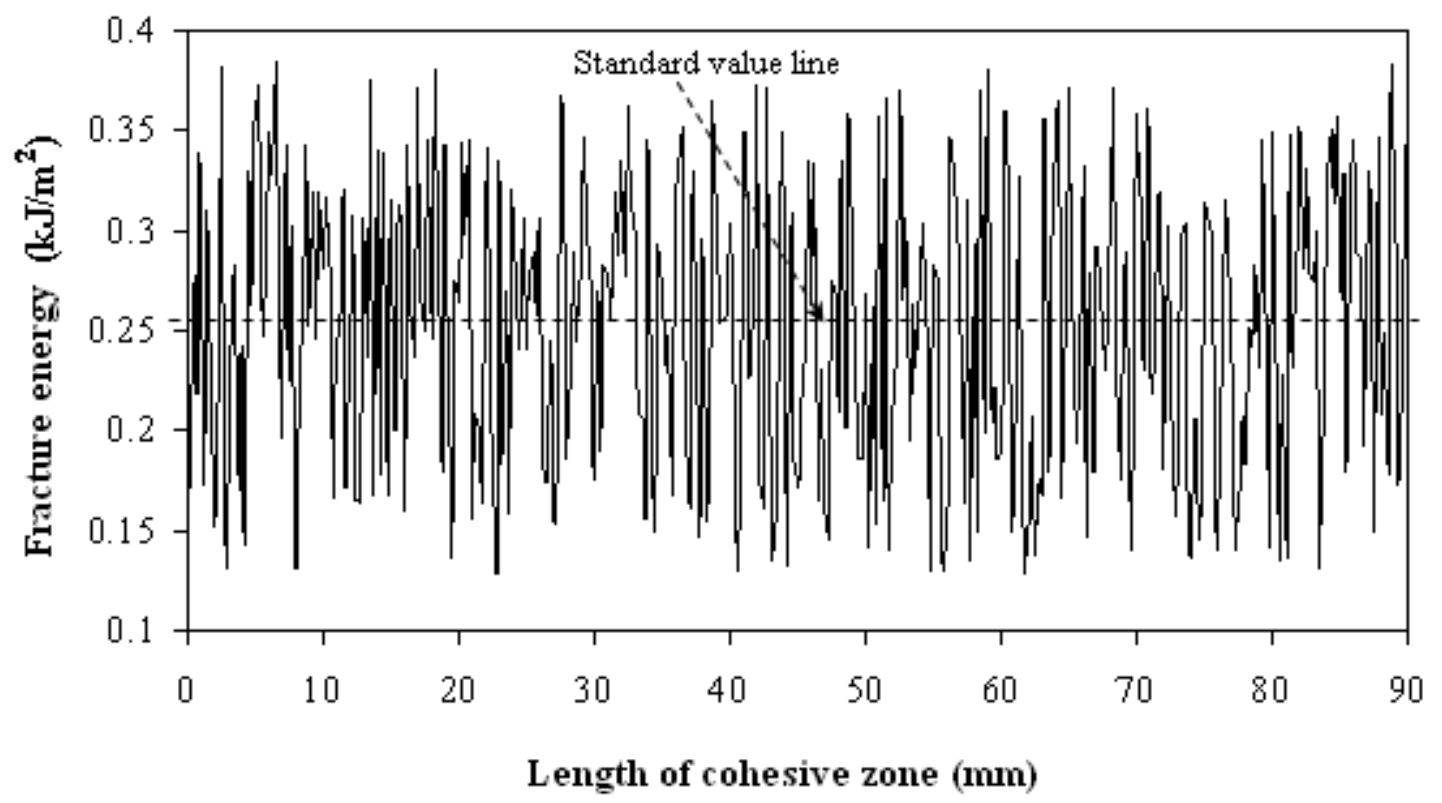

Fig. 11 Random generated data for Weibull’s distribution

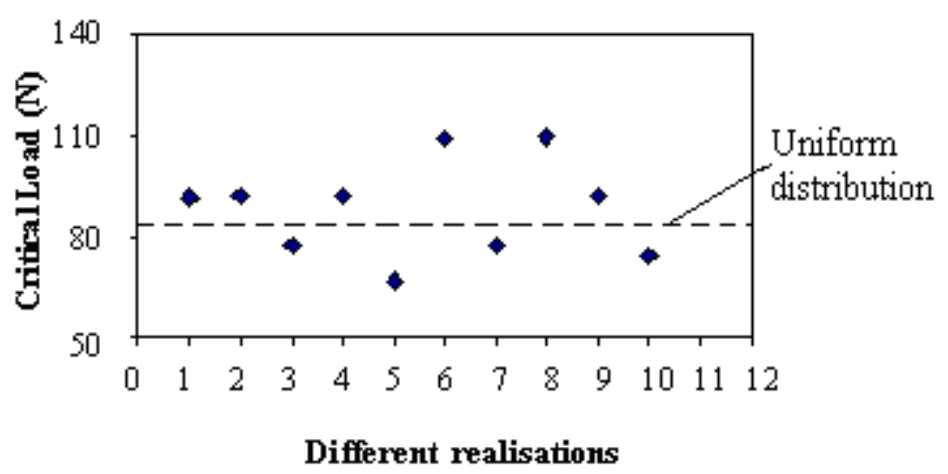

(a)

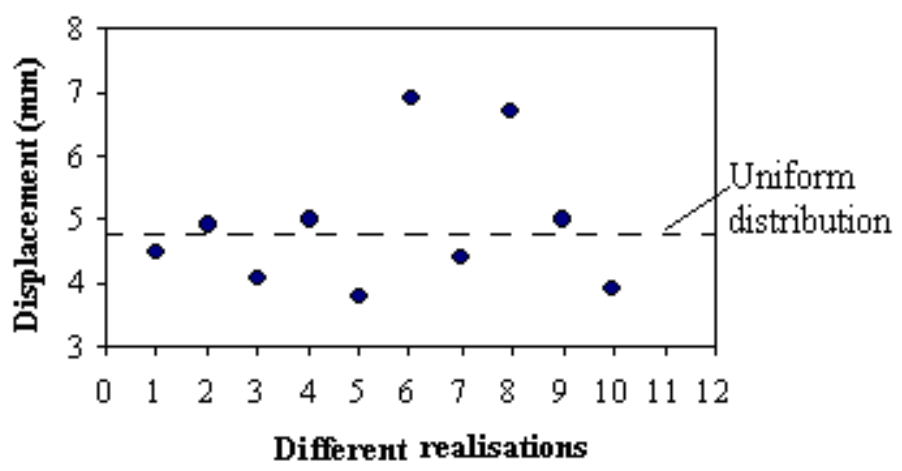

(b)

Fig. 12 Spread of (a) critical load and (b) critical displacement obtained with Method 3 as CO 